American Journal of Applied Sciences 4 (9): 645-652, 2007

ISSN 1546-9239

(C) 2007 Science Publications

\title{
Design, Fabrication, and Testing of a Membrane Piezoelectric Tactile Sensor with Four Sensing Elements
}

\author{
${ }^{1}$ Ashkan Mirbagheri, ${ }^{1}$ Javad Dargahi, ${ }^{2}$ Siamak Najarian, ${ }^{3}$ Farhad Tabatabai Ghomshe \\ ${ }^{1}$ Concordia University, Department of Mechanical and Industrial Eng., 1455 de Maisonneuve \\ Blvd. West, Montreal, Quebec, Canada \\ ${ }^{2}$ Department of Biomechanics, Artificial Tactile Sensing and Robotic Surgery Lab, Faculty of \\ Biomedical Engineering, Amirkabir University of Technology, Tehran, Iran \\ ${ }^{3}$ University of Social Welfare \& Rehabilitation Sciences, Tehran, Iran
}

\begin{abstract}
We presented design, fabrication, and testing of a novel membrane tactile sensing system with only four sensing elements. By using membrane stress combined with triangulation approach, it was possible to overcome the problems such as cross-talk between sensing elements, fragility, and complexity. The fabrication of the sensing elements performed using photolithographic and etching techniques. Position, orientation, and magnitude of applied loads through various flat shaped probes can be determined, by using only four sensing elements.
\end{abstract}

Keywords: Tactile Sensing, Minimally Invasive Surgery

\section{INTRODUCTION}

The fundamental requirement of a competent tactile sensor for manipulating an object is to determine the magnitude and the position of an applied force on it [1]. In addition, it is important to determine orientation of the object in relation to the tactile sensor ${ }^{[2]}$. In order to achieve these goals, most investigators have attempted to design a tactile sensor using an array of sensing elements arranged in matrix form ${ }^{[3]}$. When an object is pressed against these sensors, the output voltages from individual sensing elements represent the magnitude of the applied force and the number of sensing elements pressed indicates the position and orientation of the object in relation to the tactile sensor. There are several problems associated with this type of tactile sensors ${ }^{[4]}$. These problems include cross-talk between sensing elements, fragility, and complexity.

Tactile sensing is the process of determining physical properties through contact with objects. Tactile sensors offer exciting possibilities for use in mechatronic devices and instrumentation in many areas of science and engineering. Bio-robotics and industrial automation are the application areas that have generated the most interest because contact interactions are a fundamental feature of any physical manipulation system. However, there are many other potential application areas including minimally invasive surgery (MIS), agriculture, food processing, dentistry, entertainment, and future domestic and service industries. Therefore, the dependence on tactile sensing and its resulting data processing will become more prominent.

Among the above applications, minimally invasive surgery (MIS) is the procedure that can benefit immensely from tactile sensing and is now being widely used as one of the most preferred choices for various types of operations ${ }^{[5]}$. In MIS, any inhibitions on the surgeon's sensory abilities might lead to undesirable results ${ }^{[6-9]}$. MIS has many advantages; however, it decreases the sensory perception of the surgeon and the surgeon might accidentally cut or incur damage to some of the tissues ${ }^{[10-13]}$. This effect is more pronounced when the surgeon approaches a target tissue while moving past other healthy tissues. This could happen during grasping or manipulation of biological tissues such as veins, arteries, bones, etc. ${ }^{[14-}$ ${ }^{17]}$. Therefore, tracking of instruments during a surgical operation is being used more and more frequently to increase precision, reduce the risk of injury, plan optimal access routes preoperatively, find and follow the instruments intraoperatively, and finally, to increase

Corresponding Author: $\quad$ Siamak Najarian, Professor of Biomedical Engineering, Department of Biomechanics, Artificial Tactile Sensing and Robotic Surgery Lab, Faculty of Biomedical Engineering, Amirkabir University of Technology, Tehran, Iran, P.O. Box 15875-4413. Tel: (+98-21)-6454-2378. Fax: (+98-21)-6646-8186 
the quality of interventional procedures ${ }^{[18,19]}$. In certain areas of MIS, such as neurosurgery, the procedure is already well established, whereas in other procedures, such as laparoscopy or other endoscopic surgeries, the research activities are very limited ${ }^{[20,21]}$.

Considering the above facts and requirements, the design, fabrication, and testing of a novel membrane tactile sensing system with only four sensing elements is being presented in this research work. It is demonstrated that the magnitude of the applied load and its position on the surface of the sensor could be obtained using membrane stresses and triangulation approach with reasonable accuracy and without any noticeable cross-talk. The designed sensor can be potentially integrated with a medical probe or with robot's fingers in order to provide tactile mapping.

\section{MATERIALS AND METHODS}

Design of sensor: The sensor is comprised of a PVDF film, two square-shaped Plexiglas plates and four aluminum square-shaped sensing elements (Fig. 1). The Plexiglas plates have a side of $120 \mathrm{~mm}$ and an inner circle with a radius of $45 \mathrm{~mm}$. The central circle is the area of the PVDF film used for sensing. In other words, the PVDF film is exposed to external forces only within this circle. The thickness of the PVDF film is $25 \mu \mathrm{m}$.

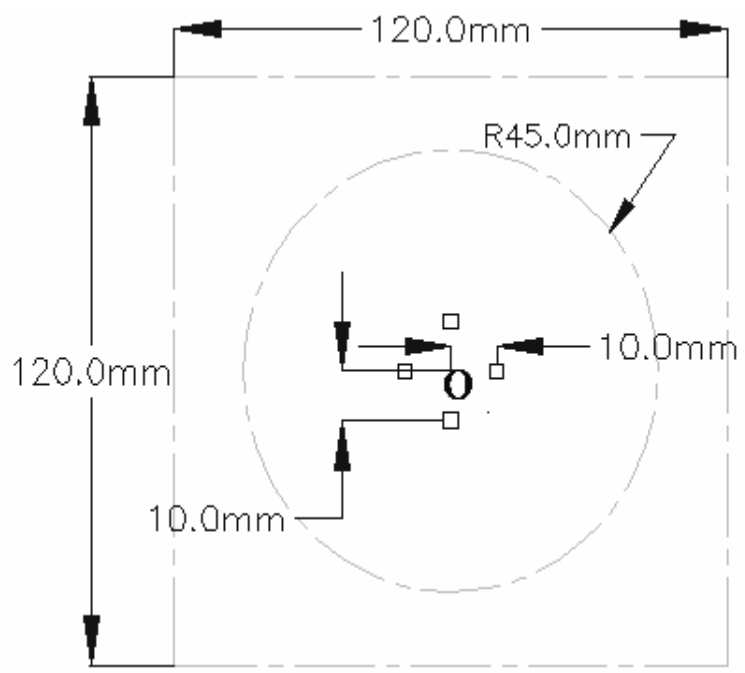

Fig. 1: Design of sensor

The sensor design also includes four sensing elements made of aluminum. Each of them is a $3 \mathrm{~mm} \times 3$ $\mathrm{mm}$ square. The center of each sensing element is located $10 \mathrm{~mm}$ away from the center of the circular area, which is denoted by the letter O in Fig. 1.
After performing the micro-fabrication and photolithography procedures on the PVDF film, the finished sensor assembly was clamped between two Plexiglas plates into each of which a circular hole was drilled all the way throughout their thicknesses. This way a constrained circular membrane was obtained. The micro-fabrication and photolithography processes are detailed below in a number of steps. The photolithography process involved seven steps: surface preparation, coating (spin coating), pre-baking (soft baking), mask alignment and exposure, photo resist removal, post-baking (hard baking), and etching.

In surface preparation process, the PVDF film was glued onto a big silicon wafer (with a radius of $45 \mathrm{~mm}$ ) using the photo resist (PR). A bare silicon wafer was first cleaned by using both acetone and de-ionized water and the photo resist was spin coated on it. Then, the pre-cut $100 \mathrm{~mm}$ square PVDF film was placed over the silicon wafer and pressed such that the PVDF film became glued to the silicon wafer substrate. In order to glue the film uniformly, a roller was used to remove the wrinkles and warps as much as possible. Then, the silicon substrate along with the PVDF was soft baked for 30 seconds at $70^{\circ} \mathrm{C}$ on a hot plate. This ensured adhesion of the PVDF film to the silicon substrate.

In spin coating process, the silicon wafer together with the PVDF film was held on a spinner chuck by vacuum. Then, the photo resist was dropped onto the surface of the PVDF film. To create a photo resist coating of uniform thickness on the PVDF film, the film and the silicon wafer were spun. Typically, the spinning rate was in the range of 3000 to $6000 \mathrm{rpm}$, and the spinning period was 15 to 30 seconds. In this research, which was partially conducted in the microfabrication laboratory of Ecole Polytechnic (based in Montreal, Canada), the Shipley S1813 photo resist was used. The thickness of the photo resist was determined primarily by its viscosity and by the spinning rate of the spinner.

In pre-baking (soft baking), the main object was to evaporate the coating solvent and to make the photo resist denser after spin coating. Typical thermal cycles were 40 minutes at $50^{\circ} \mathrm{C}$ in an oven and 45 seconds somewhere between $75^{\circ}$ to $85^{\circ} \mathrm{C}$ on a hot plate. The mask was then aligned and the film was exposed to the ultraviolet rays as is explained in the next section.

For mask alignment and exposure process, the design layout is illustrated in Fig. 2 which shows one layer of mask on each side of the film. The rectangular areas in solid lines were formed on one side of the film and those areas confined by the dashed lines were formed on the other and overlap as shown. 


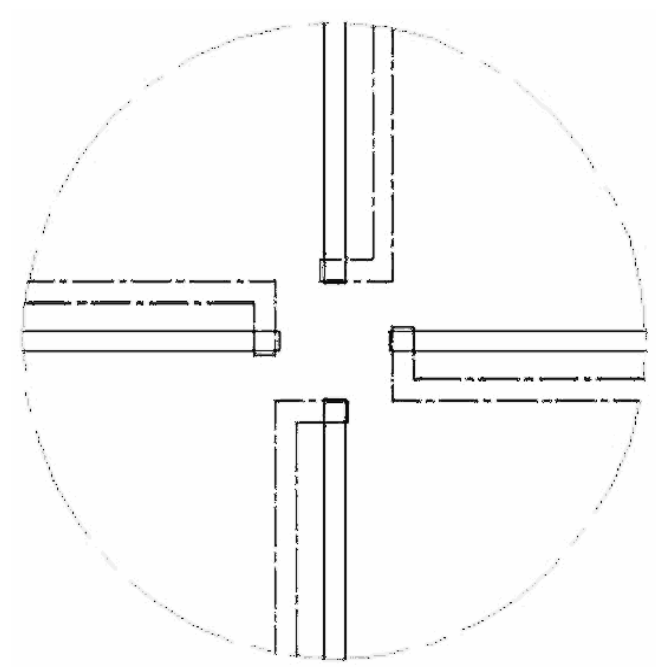

Fig. 2: Mask layout for patterning

Initially, both of the masks were drawn using AutoCAD; then, they were transferred onto two separate transparent sheets using positive or light-field masks upon which the areas of electrodes were marked as dark areas. When positive or light-field masks were exposed to ultraviolet light, rays passed only through the light areas. This resulted in polymerization of the exposed areas and helped remove them later during the etching processes. The overlap of each set of solid and dashed areas created a $3 \mathrm{~mm}$ by $3 \mathrm{~mm}$ square which was the desired electrode. Because the ultraviolet equipment was unable to expose both sides of the film at the same time, the exposure was done one side at a time.

As for the alignment, the masks were designed and fabricated to have outer dimensions of $120 \mathrm{~mm}$ by 120 $\mathrm{mm}$. The exposure time was approximately 7 seconds.

In photo resist removal, the exposed film along with its silicon wafer substrate, were immersed in a developer solution. As a result, the polymerized photo resist areas were removed. Afterwards, the film was rinsed in the de-ionized water and dried up.

In post-baking process, we removed any remaining traces of the coating solvent or developer. This was done at $50^{\circ} \mathrm{C}$ for 30 to 40 minutes in an oven or at $70^{\circ} \mathrm{C}$ for about one minute on a hot plate.

In etching process, once the photo resist was removed, the PVDF sample was dipped in a commercial aluminum etchant, transene aluminum etchant $\mathrm{A}$, which etched the aluminum on the PVDF film isotropically without affecting the PVDF. The etching was done at the room temperature. The PVDF film was dipped into the aluminum etchant and left for about three to four minutes. Finally, the sample was removed from the etchant, rinsed first in acetone, then in de-ionized water, and dried using nitrogen gas.

Experimental setup: The schematic diagram of the experimental measurement setup, developed to analyze the performance of the sensor, is shown in Fig. 3. The components of the setup in the above figure are: APower Amplifier; E-Computer; B-Signal Generator; FForce Transducer; C-Vibrator; G-Probe; D-A/D Converter and $\mathrm{H}-$ Sensor.

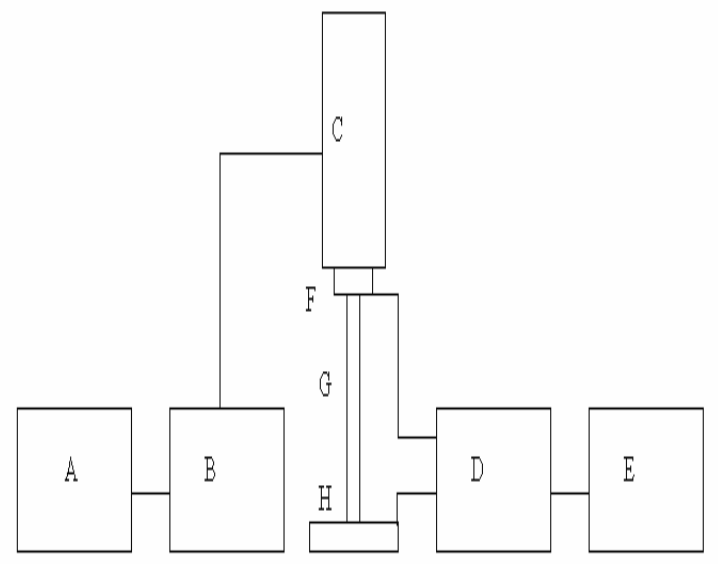

Fig. 3: Experimental set-up

The probe was driven by a vibration unit (Ling dynamic model V203) that generated a sinusoidal force having a magnitude of $1 \mathrm{~N}$ and an excitation frequency of $20 \mathrm{~Hz}$ which was transferred to the sensor assembly through the probe. The vibration unit was activated by a signal generator (Agilent 33220A model). The magnitude of the applied force was measured using a force transducer (Kistler, Type 9712B50 model), inserted between the probe and the vibration unit. The generated charges by all of the four sensing elements were measured and monitored on the computer by LabVIEW 7.0 software. The output of each sensing element was displayed on a separate channel. Hence, there were a total of four channels for monitoring the generated charges and an extra channel for monitoring the variation of the applied force. The force transducer ratio was $225 \mathrm{mV} / \mathrm{N}$. Two probes were used throughout the experiments. These were, respectively, an equilateral triangle with a side of $7 \mathrm{~mm}$ and a rectangular probe with dimensions of $14 \mathrm{~mm}$ by $7 \mathrm{~mm}$. A dynamic sinusoidal load was applied to test each sensor. The peak to peak voltages, output charges and frequency value from all of the four channels were 
captured by the data acquisition and interface system. The fabricated assembly is shown in Fig. 4.

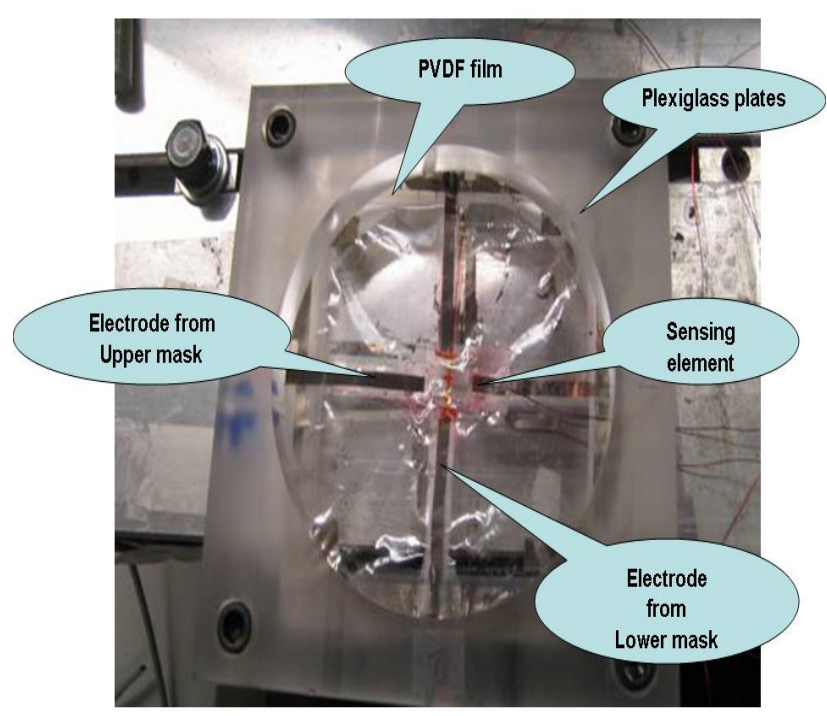

Fig. 4: Photograph of the fabricated assembly

The complete experimental set up with all the electronic components and the display unit are shown in Fig. 5.

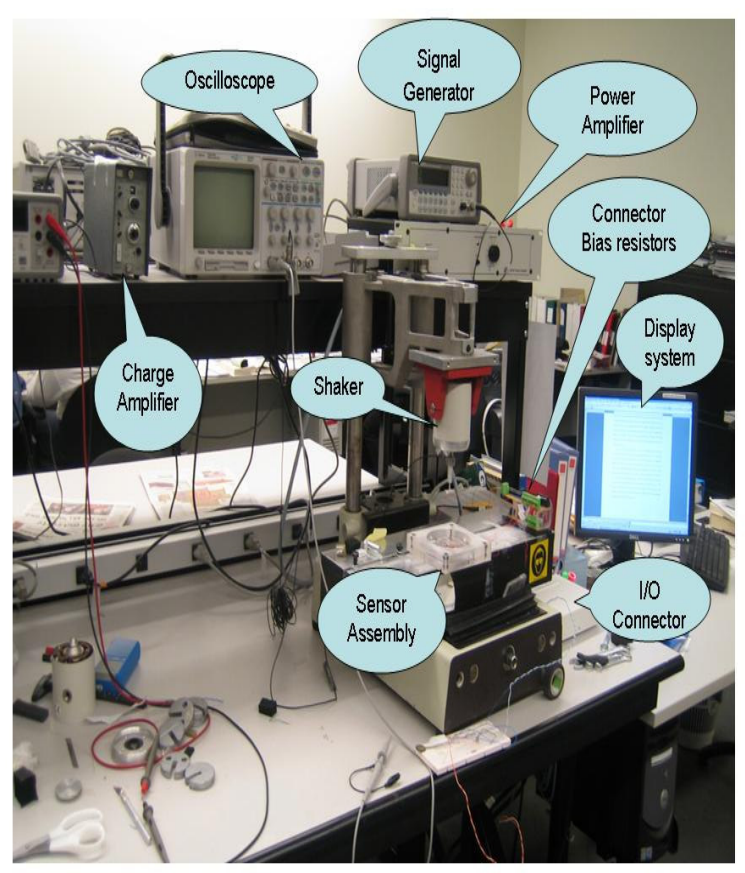

Fig. 5: Photograph of the complete experimental setup
The tactile sensor was placed under the aluminum probe. Then, a dynamic sinusoidal force was applied by the shaker which was activated by the power amplifier and the signal generator. Following this, the output from the sensor was fed to the connector box via the bias resistors. Finally, the data was then transferred to the data acquisition card inside the computer.

\section{RESULTS AND DISCUSSION}

Using the described setup, tests were conducted with probes of different shapes, sizes and orientations. Throughout the entire tests, the magnitude of force was kept at $1 \mathrm{~N}$ and the frequency stayed at a constant value of $20 \mathrm{~Hz}$.

\section{Triangular probe}

An equilateral triangular probe of $7 \mathrm{~mm}$ on each side was first selected to exert a sinusoidal load to the membrane. In Fig. 6, the obtained data corresponding to three different angles, $35^{\circ}, 65^{\circ}$, and $90^{\circ}$, are shown by markers.

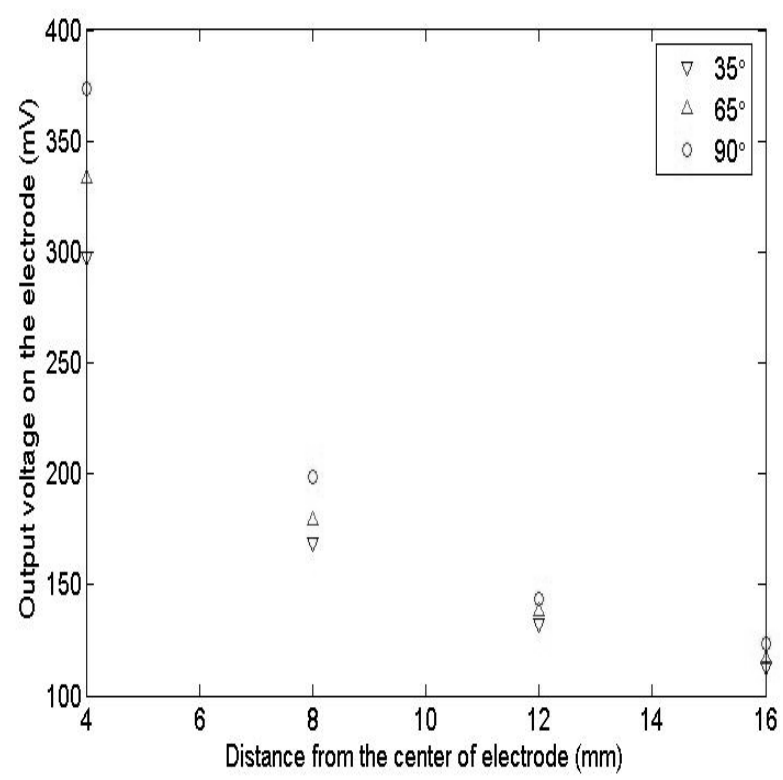

Fig. 6: Three sets of charge versus distance data for an electrode using a triangular probe.

Putting the data for all angles resulted in a crowded figure, in which it was not possible to distinguish between different sets of data. The following curves in Fig. 7 show typical voltage versus distance variations for an electrode for more angles. 


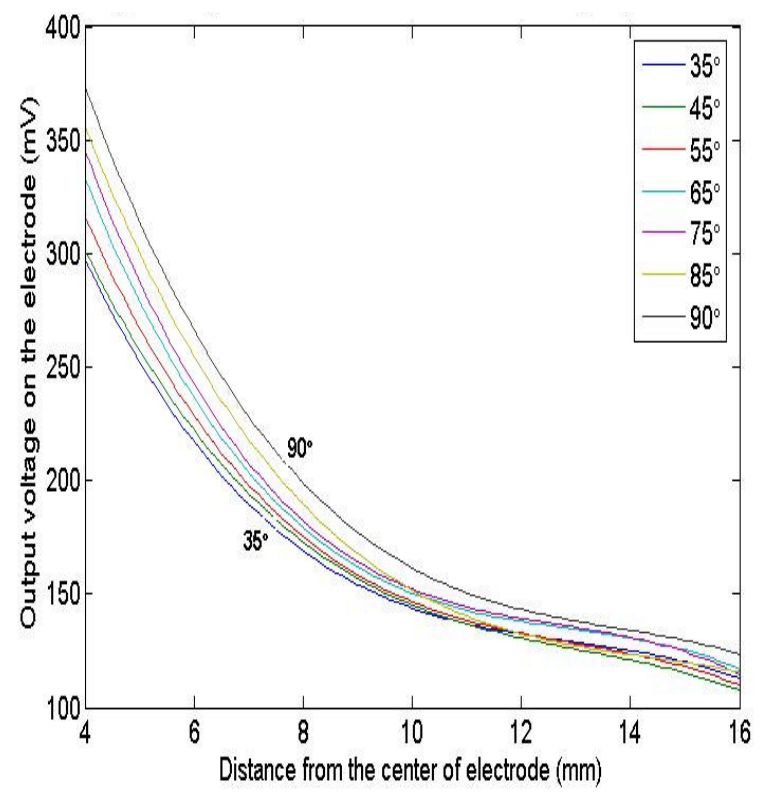

Fig. 7: Typical charge versus distance variation for an electrode using a triangular probe

Some sample triangulations and their results are presented here. In the first test, as shown in Fig. 8, the probe was put at $16 \mathrm{~mm}$ and $75^{\circ}$ with respect to the center of electrode D.

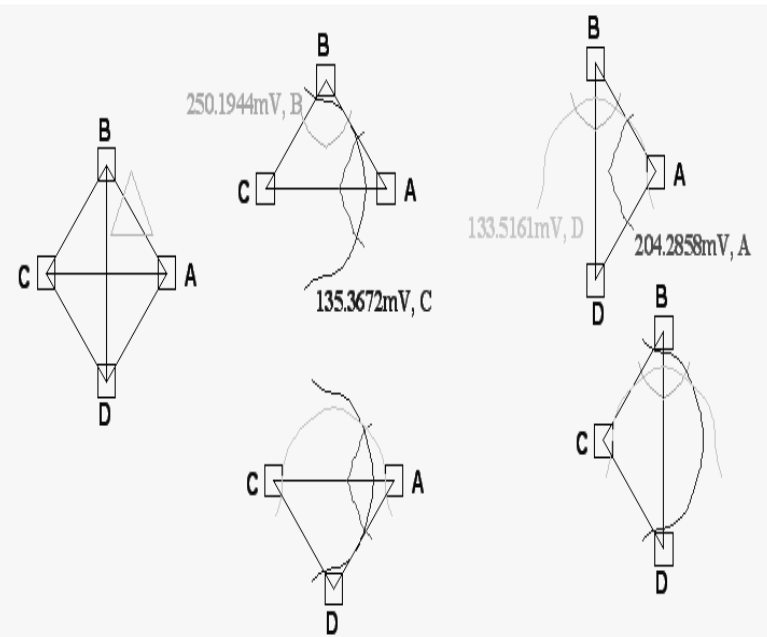

Fig. 8: First set of isocharge contours obtained from experiments

The positioning error was $0.372 \mathrm{~mm}$ which is acceptable. In the second test, the probe was put at 8 $\mathrm{mm}$ and $135^{\circ}$ with respect to the center of electrode D and rotated $90^{\circ}$ counterclockwise. This is shown in Fig. 9.
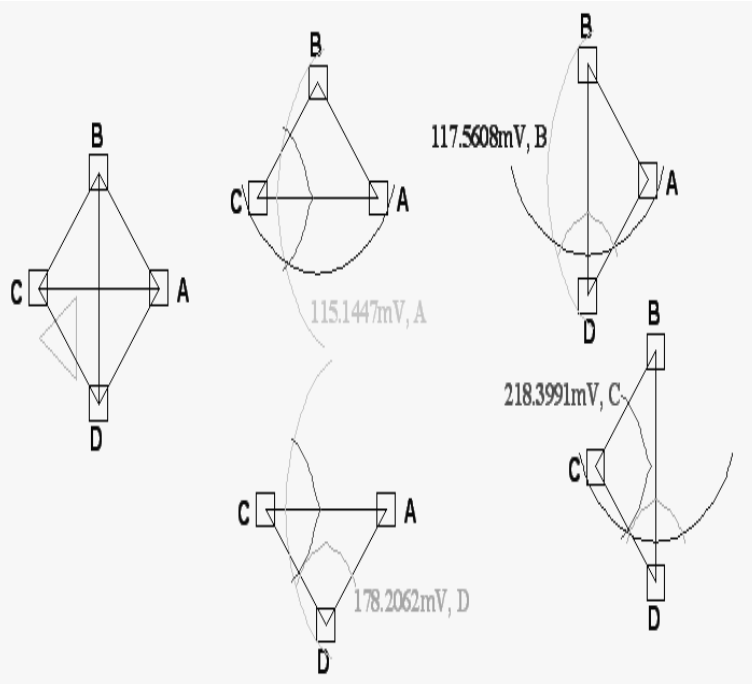

Fig. 9: Second set of experimental isocharge contours

The positioning error was calculated to be 1.411 $\mathrm{mm}$. Although greater than $1 \mathrm{~mm}$, it is still acceptable. The results for the previous two tests and three more are summarized in Table 1.

Table 1:Summary of experimental positioning results for a triangular probe.

\begin{tabular}{|c|c|}
\hline Position of the probe centroid & $\begin{array}{l}\text { Positioning error } \\
\text { for the three } \\
\text { electrode sensor } \\
(\mathrm{mm})\end{array}$ \\
\hline $\begin{array}{l}\quad 8 \mathrm{~mm}, 65^{\circ} \\
\text { (with respect to the center of } \\
\text { lower-most electrode) }\end{array}$ & 2.23 \\
\hline $\begin{array}{l}16 \mathrm{~mm}, 75^{\circ} \\
\text { (with respect to the center of } \\
\text { lower-most electrode) }\end{array}$ & 0.372 \\
\hline $\begin{array}{l}\quad 8 \mathrm{~mm}, 135^{\circ} \\
\text { (with respect to the center of } \\
\text { lower-most electrode), probe } \\
\text { rotated } 90^{\circ} \mathrm{CCW}\end{array}$ & 1.411 \\
\hline $\begin{array}{l}12 \mathrm{~mm}, 105^{\circ} \\
\text { (with respect to the center of } \\
\text { lower-most electrode), probe } \\
\text { inverted }\end{array}$ & 1.89 \\
\hline $\begin{array}{c}\text { Probe at the center, rotated } 90^{\circ} \\
\text { CW }\end{array}$ & 0.521 \\
\hline
\end{tabular}

\section{Rectangular probe}

A rectangular probe of $14 \mathrm{~mm}$ by $7 \mathrm{~mm}$ dimensions was selected as the next probe for applying 
a sinusoidal force to the membrane. In Fig. 10, the obtained data corresponding to three different angles, $35^{\circ}, 65^{\circ}$, and $90^{\circ}$, are shown by markers.

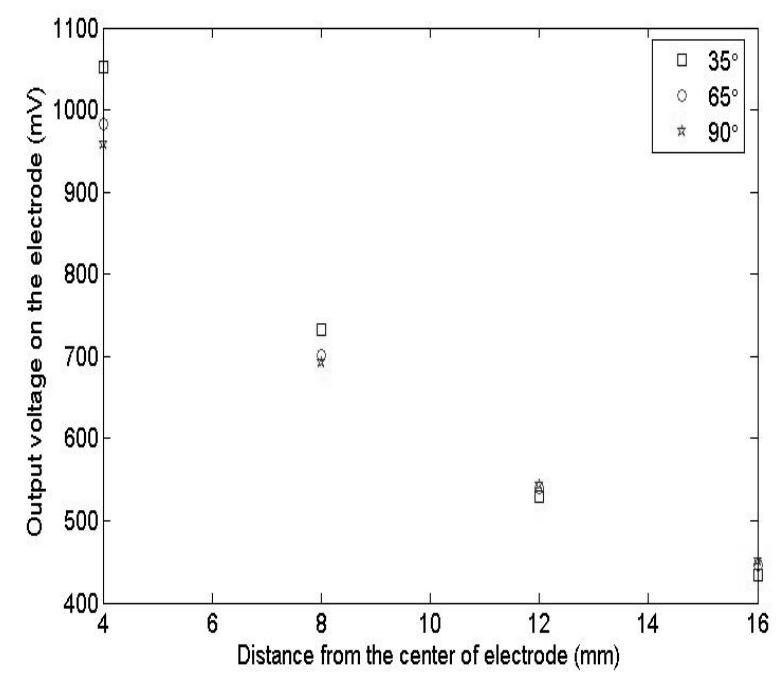

Fig. 10: Three sets of charge versus distance data for an electrode using a rectangular probe

Similar to the triangular probe, since putting the data for all angles resulted in a crowded figure, only three sets of data were displayed in the previous figure. The following curves show typical voltage versus distance variations for an electrode (Fig. 11).

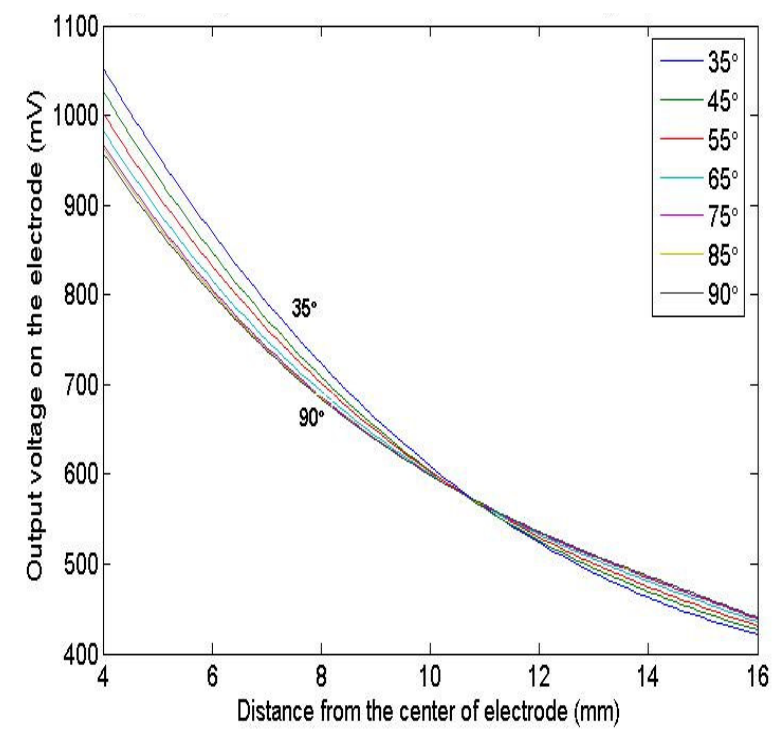

Fig. 11: Typical charge versus distance variation for an electrode using a rectangular probe

A number of tests were performed, this time with the rectangular probe. In the first experiment, as shown in Fig. 12, the probe was put at $16 \mathrm{~mm}$ and $75^{\circ}$ with respect to the center of electrode $\mathrm{D}$.

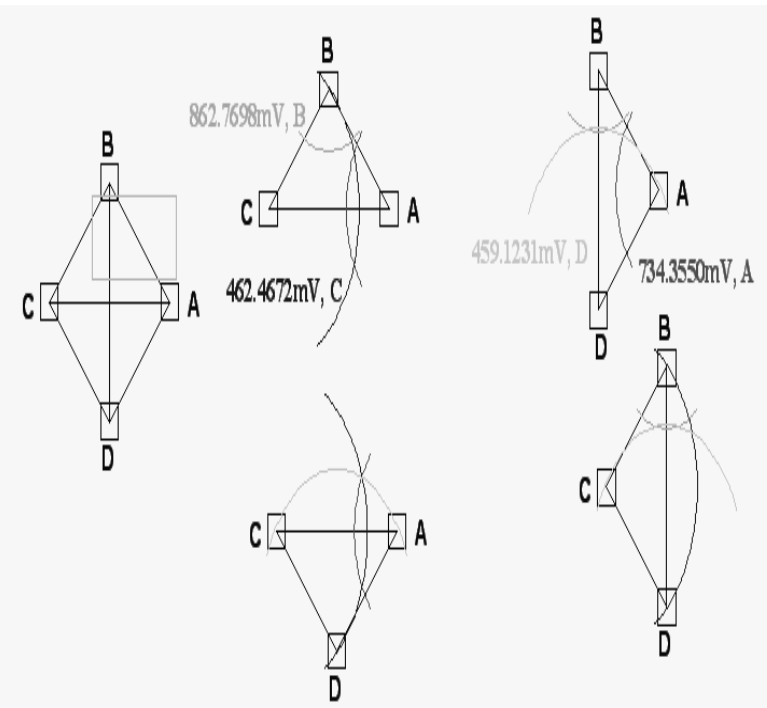

Fig. 12: Results of first test with a rectangular probe

The positioning error is $0.764 \mathrm{~mm}$ which is acceptable. In the second test with a rectangular probe, the probe was put at $8 \mathrm{~mm}$ and $135^{\circ}$ with respect to the center of electrode $\mathrm{D}$ and rotated $90^{\circ}$ counterclockwise (Fig. 13).

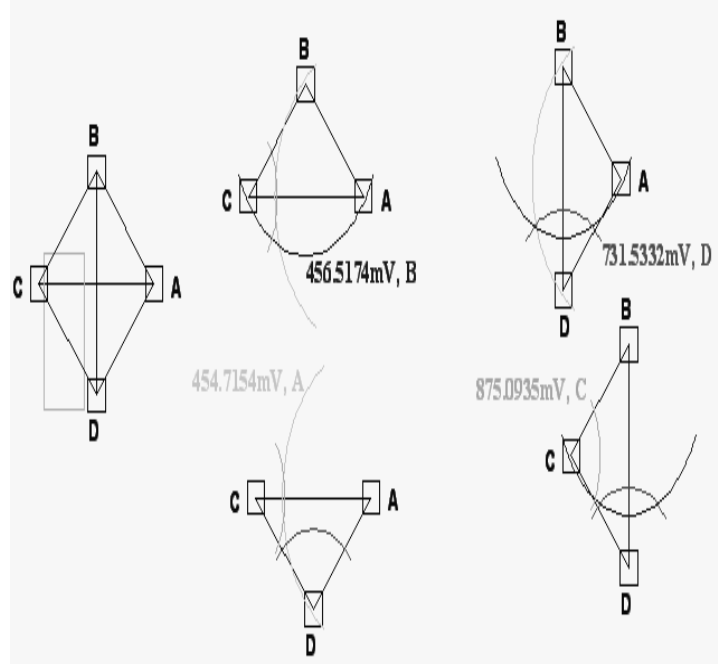

Fig. 13: Results of second test with a rectangular probe.

The positioning error was calculated to be 0.792 $\mathrm{mm}$ which falls below $1 \mathrm{~mm}$ and is acceptable. The results for the previous two tests and three more are summarized in Table 2. 
Table 2: Summary of experimental positioning results for a rectangular probe.

\begin{tabular}{|c|c|}
\hline Position of the probe centroid & $\begin{array}{c}\text { Positioning } \\
\text { error for the } \\
\text { three electrode } \\
\text { sensor }(\mathbf{m m})\end{array}$ \\
\hline $8 \mathrm{~mm}, 65^{\circ}$ & \\
\hline $\begin{array}{l}\text { (with respect to the center of } \\
\text { lower-most electrode) }\end{array}$ & 1.325 \\
\hline $16 \mathrm{~mm}, 75^{\circ}$ & \\
\hline $\begin{array}{l}\text { (with respect to the center of } \\
\text { lower-most electrode) }\end{array}$ & 0.764 \\
\hline $8 \mathrm{~mm}, 135^{\circ}$ & \\
\hline $\begin{array}{l}\text { (with respect to the center of } \\
\text { lower-most electrode), probe } \\
\text { rotated } 90^{\circ} \mathrm{CCW}\end{array}$ & 0.792 \\
\hline $12 \mathrm{~mm}, 105^{\circ}$ & \\
\hline $\begin{array}{l}\text { (with respect to the center of } \\
\text { lower-most electrode), probe } \\
\text { rotated } 45^{\circ} \mathrm{CW}\end{array}$ & 0.924 \\
\hline $\begin{array}{l}\text { Probe at the center, rotated } 90^{\circ} \\
\mathrm{CW}\end{array}$ & $\begin{array}{l}\text { No intersection } \\
\text { of contours }\end{array}$ \\
\hline
\end{tabular}

\section{CONCLUSION}

It can be concluded that when it comes to detecting forces which are applied via non-circular probes, a four-electrode biaxial sensor is the right expediency. For forces with magnitudes other than $1 \mathrm{~N}$, at least in the band with an upper limit of $2 \mathrm{~N}$, the triangulation method always works for detecting the position of the applied force. Output electrical charges of the sensor vary linearly against the magnitude of the applied force up to $0.5 \mathrm{~N}$. For magnitudes of forces above this value, although the charge vs. force variation becomes nonlinear, it is still possible to approximate the magnitude of the applied force. When using a uniaxial film, either with three- or four- electrodes, the system is not effective and reliable enough for detecting centroids of objects or positions of the applied forces when the exerted force is not a concentrated force, or when the shape of the probe is non-circular. Increasing the thickness of the film has a less than desired effect on the linearity of the system. Moreover, it introduces a third source for generation of electrical charge, along the 3- or thickness direction of PVDF film, which adds complexity and further jeopardizes the linearity of the system.

The sensor fabrication problem was a big challenge because of its scales and dimensions. Although the facilities and equipment in Ecole Polytechnic's microfabrication lab were quite adequate, the authors had to make several tries and errors in order to achieve the appropriate parameters involved. Eventually, the fabricated sensor was close enough to the desired pattern.

An important factor that we are considering for our future works is the pyroelectric response of the PVDF film. In practice, when an object like a biological tissue touches the PVDF film, it does not necessarily have the same temperature as that of the PVDF film and its sensing elements. This temperature differential can result in abrupt and noticeable outputs from the sensor. Another point to work on for the future is enabling the sensor to detect the shear stress components.

\section{ACKNOWLEDGEMENTS}

The authors thank the Institute for Robotics and Intelligent Systems (IRIS), and the Natural Sciences and Engineering Research Council (NSERC) of Canada for providing partial financial support. We also appreciate the support provided via the grant awarded by the Ministry of Health of Iran through Razi International Festival. We also would like to express our gratitude to the Center of Excellence of Biomedical Engineering of Iran based in Amirkabir University of technology, Faculty of Biomedical Engineering for its contribution.

\section{REFERENCES}

1. Dario, P., 1991. Tactile Sensing-Technology and Applications. Sensors and Actuators A-Physical, 26(1/3): 251-261.

2. Dargahi, J., S. Najarian, 2004. Analysis of a Membrane Type Polymeric-Based Tactile Sensor for Biomedical and Medical Robotic Applications. Sensors and Materials, 16(1): 25-41.

3. Dargahi, J., S. Najarian, 2004. An Integrated Force-Position Tactile Sensor for Improving Diagnostic and Therapeutic Endoscopic Surgery. Bio-Medical Materials and Engineering, 14(2): 151-166.

4. Dargahi, J., 1998. Piezoelectric and Pyroelectric Transient Signal Analysis for Detecting the Temperature of an Object for Robotic Tactile Sensing. Sensor and Actuators A-Physical, 71(1/2): 89-97.

5. Dargahi, J., S. Najarian, 2004. Theoretical and Experimental Analysis of a Piezoelectric Tactile Sensor for Use in Endoscopic Surgery. Sensor Review, 24(1): 74-83. 
6. Fischer, H., B. Neisius, R. Trapp, 1995. Interactive Technology and a New Paradigm for Health Care. IOS Press, The Netherlands.

7. Bicchi, A., G. Canepa, D.De. Rossi, P. Iacconi, E.P. Scilingo, 1996. A Sensorized Minimally Invasive Surgery Tool for Detecting Tissue Elastic Properties. Proceedings of IEEE International Conference Robotics and Automation, Minneapolis, USA, 884-888.

8. Brouwer, I., J. Ustin, L. Bentley, A. Sherman, N. Dhruv, F. Tendick, 2001. Measuring in vivo Animal Soft Tissue Properties for Haptic Modeling in Surgical Simulation. In Studies in Health Technology Informatics - Medicine Meets Virtual Reality, Amsterdam: ISO Press, 69-74.

9. Dargahi, J., S. Najarian, 2004. A Supported Membrane Type Sensor for Medical Tactile Mapping. Sensor Review, 24(3): 284-297.

10. Hannaford, B., J. Trujillo, M. Sinanan, M. Moreyra, J. Rosen, J. Brown, R. Leuschke, M. MacFarlane, 1998. Computerized Endoscopic Surgical Grasper. In Studies in Health Technology Informatics-Medicine Meets Virtual Reality, Amsterdam, ISO Press, 265-271.

11. Dargahi, J., S. Najarian, 2004. Human Tactile Perception as a Standard for Artificial Tactile Sensing-a review. International Journal of Medical Robotics and Computer Assisted Surgery, 1(13): 23-35.

12. Dargahi, J., S. Najarian, 2005. Advances in Tactile Sensors Design/Manufacturing and Its Impact on Robotics Applications- a review. Industrial Robot, 32(3): 268-281.

13. Dargahi, J., S. Najarian, 2003. An Endoscopic Force Position Grasper with Minimum Sensors. Canadian Journal of Electrical and Computer Engineering, 28(3/4): 155-161.
14. Dargahi, J., S. Najarian, X.Z. Zheng, 2005. Measurements and Modeling of Compliance Using a Novel Multi-Sensor Endoscopic Grasper Device. Sensors and Materials, 17(1): 7-20.

15. Lee, M.H., H.R. Nicholls, 1999. Tactile Sensing for Mechatronics-A State-of-the-Art Surgery. Mechatronics, 9(1): 1-31.

16. Bar-Cohen, Y., C. Mavroidis, M. Bouzit, B. Dolgin, D. Harm, G. Kopchok, R. White, 2000. Virtual Reality Robotic Operation Simulations Using MEMICA Haptic System. Proc. Int. Conf. for Smart Systems and Robotics for Medicine and Space Applications, Houston, USA.

17. Fisch, A., C. Mavroidis, J. Melli-Huber, Y. BarCohen, 2003. Haptic Devices for Virtual Reality, Telepresence, and Human-Assistive Robotics. chap. 4 in Biologically-Inspired Intelligent Robots, Bellingham, Wash.

18. Dargahi, J., M. Parameswaran, S. Payandeh, 2000. A Micromachined Piezoelectric Tactile Sensor for Endoscopic Grasper-Theory, Fabrication, and Experiments. Journal of Microelectromechanical Systems, 9(3): 329-335.

19. Burger, F., P.A. Besse, R.S. Popvic, 1998. New Fully Integrated 3D Silicon Hall Sensor for Precise Angular-Position Measurements. Sensors and Actuators A, 67: 72-76.

20. Dargahi, J., 2000. A Three Sensing Element Piezoelectric Tactile Sensor for Robotic and Prosthetic Applications. Sensors and Actuators APhysical, 80(1): 23-30.

21. Dargahi, J., 2002. An Endoscopic and Robotic Tooth-Like Compliance and Roughness Tactile Sensor. Journal of Mechanical Design, 124: 576582. 